\title{
Fuzzy Linear Programming: Optimization of an Electric Circuit Model
}

\author{
A.M.A. BERTONE, R.S.M. JAFELICE and M.A. CÂMARA*
}

Received on November 19, 2016 / Accepted on June 16, 2017

\begin{abstract}
A problem of a voltage division circuit is modeled in order to determine the values of the resistors centered, in a way that the impedance of the resistance voltage divider is minimal. This problem is equivalent to maximizing the admittance, associated to the resistance, which is defined as the quotient of the electric current and its voltage, measured in Siemens. Three cases are analyzed for the components of the linear programming: real numbers, fuzzy numbers of type-1, and fuzzy sets of type-2. The first case is considered in order to validate the other two cases. The optimal solution in the fuzzy linear programming of type-1 is obtained through a linear defuzzification function, defined in the trapezoidal fuzzy numbers subspace of fuzzy numbers vector space, which allows to solve the corresponding linear programming problem with real components. A study upon the parameter for linear defuzzification is accomplished to determine the best representative of the family of parameters. The $\alpha$-levels representation theorem is the method to obtain the optimal solution of type-2. For each $\alpha$-level is solved a fuzzy linear programming problem of type-1, using the previous methodology. Numerical simulations illustrate the results in the three cases.
\end{abstract}

Keywords: electrical circuit model, linear programming, fuzzy linear programming, fuzzy sets of type-1, fuzzy sets of type-2.

\section{INTRODUCTION}

Being one of the most important field of Operational Research, Linear Programming (LP) concerns to models that optimize linear objective function and constraints on the decision variables. Efficient algorithms has been developed for LP, among which we point out the interior point [12], and the simplex algorithm [3].

One of the important issues of LP is that the modeling needs well-defined and precise data, which in general involves high information costs. Indeed, this is a task almost impossible to accomplish in many cases, due to risk or uncertainty in some data [9]. This problem of the classical LP can be contoured with the use of fuzzy numbers. Fuzzy Linear Programming (FLP) explains the mathematical model in a more realistic way [11]. Basically, considering some of the components

*Corresponding author: Marcos Antônio da Câmara - E-mail: camara@ufu.br

Faculdade de Matemática - FAMAT, Universidade Federal de Uberlândia, 38408-902 Uberlândia, MG, Brasil.

E-mails: amabertone@ufu.br; rmotta@ufu.br 
of a LP as fuzzy numbers, a constraint violation is allowed, and the degree of satisfaction of a constraint is defined as the membership function of the constraint [9].

One of the basic references in the area of FLP is due to Bellman and Zadeh [2]. The type of FLP that has been considered for this research is the possibilistic programming, that recognizes uncertainties in the objective function coefficients, as well as in constraint coefficients. The membership function is used to represent the degree of satisfaction of constraints, the decision-maker's expectations about the objective function level, and the range of uncertainty of coefficients [9].

Based in the great success of the FLP, the approach is applied to a voltage divider model. In fact, an electrical circuit has components characterized by diverse parameters, each one associated to a tolerance, given by the product manufacturer. For example, a resistance of $48 \mathrm{ohms}$ can have $\pm 10 \%$ tolerance, due to temperature, time of use, among other reasons [10]. This gradualness motivated this study to use the fuzzy linear programming approach for a problem of a voltage divider. The value given by the electric appliance manufacturer is called the centered value. The tolerances and the centered values allow better control over the limits under which the circuit keeps operating and, thereby, optimize its performance. The model use for this research was built by Salazar in [10]. The objective of this research is to study three cases for the components of the LP. The first case is for real numbers components we proposed of validating the other two results. The second case refers to trapezoidal fuzzy number of type- 1 as a components of LP system. The optimal solution is obtained through a total linear order defuzzification function, defined in the trapezoidal fuzzy numbers subspace of fuzzy numbers vector space. The third case extends the second case for type- 2 independent LP components. The $\alpha$-levels representation theorem is the method to obtain the optimal solution of type-2. For the numerical simulation for all cases it is use the classical interior point method [12], using the linear programming algorithm linprog of the software Matlab ${ }^{\circledR}$, and an algorithm that reproduces the representation theorem.

This work is organized into six sections. Section 1 is the introduction to this study. In Section 2 the mathematical model of the electrical circuit is explained. In Section 3 a fuzzy background for the electrical circuit is detailed. In Section 4 the fuzzy linear programming method for the model is developed. The numerical results are shown in Section 5, and conclusions are drawn in Section 6.

\section{MATHEMATICAL MODEL OF THE ELECTRICAL CIRCUIT}

The voltage divider circuit shown in [10], illustrated in Figure 1(a), is the model to be built in this section.

In this circuit are considered two voltage generators, each producing an electromotive force, $E_{1}$ and $E_{2}$, measured in volts. Suppose that $E_{1}>E_{2}$ for the circuit shown in Figure 1(a), the current in the direction indicated by the arrows. It is assumed that the tolerances associated with respective potential have a minimum value $E_{1}^{-}$, and maximum $E_{1}^{+}$, with respect to $E_{1}$. Analogously, there exist minimum value $E_{2}^{-}$, and maximum $E_{2}^{+}$, with respect to $E_{2}$. The goal of this model is determine the centered values of the resistors, $R_{1}$ and $R_{2}$, so that the impedance of 


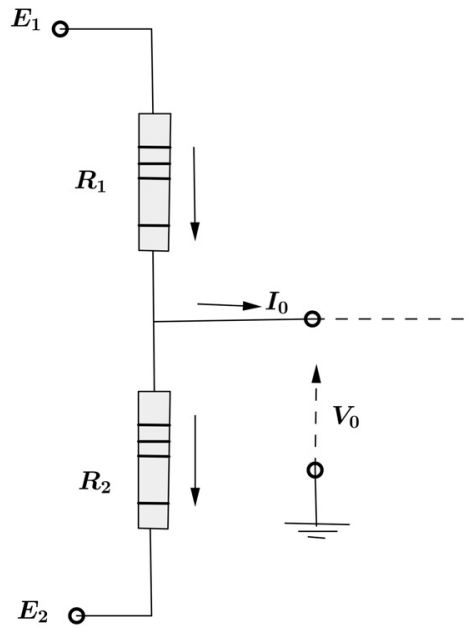

(a) The circuit model of voltage divider

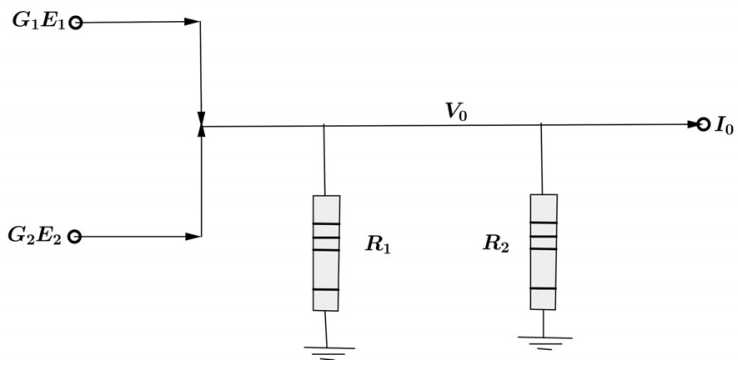

(b) Parallel circuit model of voltage divider

Figure 1: Equivalente basic circuit of voltage divider.

the resistant voltage divider is minimal. Further, as restrictions, the output potential, $V_{0}$, belongs to the interval $\left[V_{0}^{m}, V_{0}^{M}\right]$, where the exponent $m$ stands for the minimum value, and $M$, for the maximum. The current, $I_{0}$, is in the range $\left[I_{0}^{m}, I_{0}^{M}\right]$. The tolerances verify the inequalities

$$
E_{1}^{-} \geq V_{0}^{M} \geq V_{0}^{m} \geq E_{2}^{+} \text {. }
$$

The total impedance of the resistant voltage divider is given by the formula

$$
R_{0}=\frac{R_{1} R_{2}}{R_{1}+R_{2}}
$$

due to the fact that the two resistors are in parallel. In order to obtain a linear model, the variable resistors are replaced by the associated admittance, which are given by the inverse of the values $G_{i}=1 / R_{i}, i=0,1,2$. With this notation, total admittance divider $G_{0}=G_{1}+G_{2}$. As a consequence, the objective function of the problem is given by

$$
G_{0}^{-}=G_{1}^{-}+G_{2}^{-},
$$

which it will be maximized. Denoting by $G_{1}$ and $G_{2}$ the centered values of the admittance $G_{1}$ and $G_{2}$, respectively, then we have

$$
G_{1}^{-}=\left(1-\epsilon_{1}\right) G_{1} \text { and } G_{2}^{-}=\left(1-\epsilon_{2}\right) G_{2},
$$

where $\epsilon_{1}$ and $\epsilon_{2}$ are two known tolerances. Thus, the proposed linear programming problem is given by

$$
\max \left(H\left(G_{1}, G_{2}\right)\right) \text {, where } H\left(G_{1}, G_{2}\right)=\left(1-\epsilon_{1}\right) G_{1}+\left(1-\epsilon_{2}\right) G_{2} \text {. }
$$


In order to express the constraints on the variables $\mathcal{G}_{1}$ and $\mathcal{G}_{2}$, we observe, based in Figure 1(b), that

$$
I=I_{1}+I_{2}+I_{0}
$$

Consequently, we get

$$
G_{1} E_{1}+G_{2} E_{2}=\frac{V_{0}}{R_{1}}+\frac{V_{0}}{R_{2}}+I_{0}
$$

Hence,

$$
G_{1} E_{1}+G_{2} E_{2}=V_{0}\left(\frac{1}{R_{1}}+\frac{1}{R_{2}}\right)+I_{0},
$$

from where, it is obtained

$$
G_{1} E_{1}+G_{2} E_{2}-I_{0}=V_{0}\left(G_{1}+G_{2}\right)
$$

Therefore,

$$
V_{0}=\frac{E_{1} G_{1}+E_{2} G_{2}-I_{0}}{G_{1}+G_{2}} .
$$

Analyzing the behavior of $V_{0}$ in relation to the variations of $G_{1}, G_{2}$, and $I_{0}$, we have:

(I) Calculating the partial derivative of $V_{0}$ with respect to $G_{1}$, we obtain

$$
\begin{aligned}
\frac{\partial V_{0}}{\partial G_{1}} & =\frac{E_{1}\left(G_{1}+G_{2}\right)-\left(E_{1} G_{1}+E_{2} G_{2}-I_{0}\right)}{\left(G_{1}+G_{2}\right)^{2}} \\
& =\frac{E_{1} G_{1}+E_{1} G_{2}-E_{1} G_{1}-E_{2} G_{2}+I_{0}}{\left(G_{1}+G_{2}\right)^{2}} \\
& =\frac{\left(E_{1}-E_{2}\right) G_{2}+I_{0}}{\left(G_{1}+G_{2}\right)^{2}} \\
& >0,
\end{aligned}
$$

due to the fact that $E_{1}>E_{2}$. As a conclusion, we get that $V_{0}$ decreases as $G_{1}$ decreases.

(II) Calculating the partial derivative of $V_{0}$ with respect to $G_{2}$, we have

$$
\frac{\partial V_{0}}{\partial G_{2}}=\frac{\left(E_{2}-E_{1}\right) G_{1}+I_{0}}{\left(G_{1}+G_{2}\right)^{2}}<0
$$

since $I=I_{1}+I_{2}+I_{0}$ so that

$$
I_{0}=I-I_{1}-I_{2}=G_{1} E_{1}+G_{2} E_{2}-V_{0} G_{1}-V_{0} G_{2} .
$$


Thus,

$$
\begin{aligned}
\frac{\partial V_{0}}{\partial G_{2}} & =\frac{\left(E_{2}-E_{1}\right) G_{1}+I_{0}}{\left(G_{1}+G_{2}\right)^{2}} \\
& =\frac{G_{1} E_{2}-G_{1} E_{1}+G_{1} E_{1}+G_{2} E_{2}-V_{0} G_{1}-V_{0} G_{2}}{\left(G_{1}+G_{2}\right)^{2}} \\
& =\frac{E_{2}\left(G_{1}+G_{2}\right)-V_{0}\left(G_{1}+G_{2}\right)}{\left(G_{1}+G_{2}\right)^{2}} \\
& =\frac{E_{2}-V_{0}}{G_{1}+G_{2}} .
\end{aligned}
$$

Since we have $E_{1}^{-} \geq V_{0}^{M} \geq V_{0}^{m} \geq E_{2}^{+}$, then $E_{2}<V_{0}$, therefore $E_{2}-V_{0}<0$. This implies that

$$
\frac{\partial V_{0}}{\partial G_{2}}<0
$$

meaning that $V_{0}$ decreases as $G_{2}$ increases.

(III) From the fact that $G_{1}+G_{2}>0$, we conclude

$$
\frac{\partial V_{0}}{\partial I_{0}}=\frac{-1}{G_{1}+G_{2}}<0
$$

which yields $V_{0}$ decreases as $I_{0}$ increases.

Consequently, $V_{0}$ will assume its lowest value, which it is desired to be not less than $V_{0}^{m}$, when $G_{1}$ assumes its lowest value $G_{1}^{-}$. Besides, $G_{2}$ assumes its highest value, $G_{2}^{+}$, and the current obtained $I_{0}$ is $I_{0}^{M}$.

Therefore, the constraint $V_{0}^{m} \leq V_{0}$ is equivalent to

$$
V_{0}^{m} \leq \frac{E_{1}^{-} G_{1}^{-}+E_{2}^{-} G_{2}^{+}-I_{0}^{M}}{G_{1}^{-}+G_{2}^{+}}
$$

or, alternatively,

$$
V_{0}^{m} \leq \frac{E_{1}^{-}\left(1-\epsilon_{1}\right) G_{1}+E_{2}^{-}\left(1+\epsilon_{2}\right) G_{2}-I_{0}^{M}}{\left(1-\epsilon_{1}\right) G_{1}+\left(1+\epsilon_{2}\right) G_{2}}
$$

that is,

$$
\left(1-\epsilon_{1}\right)\left(E_{1}^{-}-V_{0}^{m}\right) G_{1}+\left(1+\epsilon_{2}\right)\left(E_{2}^{-}-V_{0}^{m}\right) G_{2} \geq I_{0}^{M} .
$$

Similarly, the constraint $V_{0} \leq V_{0}^{M}$ is equivalent to

$$
\left(1+\epsilon_{1}\right)\left(E_{1}^{+}-V_{0}^{M}\right) G_{1}+\left(1-\epsilon_{2}\right)\left(E_{2}^{+}-V_{0}^{M}\right) G_{2} \leq I_{0}^{m} .
$$

Consequently, the mathematical model for the linear programming for the problem of the voltage divider is given by

$$
\begin{gathered}
\max H\left(G_{1}, G_{2}\right)=\left[\left(1-\epsilon_{1}\right) \mathcal{G}_{1}+\left(1-\epsilon_{2}\right) \mathcal{G}_{2}\right] \\
\left(1-\epsilon_{1}\right)\left(E_{1}^{-}-V_{0}^{m}\right) \mathcal{G}_{1}+\left(1+\epsilon_{2}\right)\left(E_{2}^{-}-V_{0}^{m}\right) \mathcal{G}_{2} \geq I_{0}^{M} \\
\left(1+\epsilon_{1}\right)\left(E_{1}^{+}-V_{0}^{M}\right) \mathcal{G}_{1}+\left(1-\epsilon_{2}\right)\left(E_{2}^{+}-V_{0}^{M}\right) \mathcal{G}_{2} \leq I_{0}^{m}
\end{gathered}
$$


Using the notations

$$
\epsilon=\left(\begin{array}{l}
1-\epsilon_{1} \\
1-\epsilon_{2}
\end{array}\right), \quad G=\left(\begin{array}{c}
G_{1} \\
G_{2}
\end{array}\right), \quad \Upsilon=\left(\begin{array}{c}
-I_{0}^{M} \\
I_{0}^{m}
\end{array}\right), \quad \mathcal{O}=\left(\begin{array}{l}
0 \\
0
\end{array}\right)
$$

and

$$
\Theta=\left(\begin{array}{ll}
\left(1-\epsilon_{1}\right)\left(V_{0}^{m}-E_{1}^{-}\right) & \left(1+\epsilon_{2}\right)\left(V_{0}^{m}-E_{2}^{-}\right) \\
\left(1+\epsilon_{1}\right)\left(E_{1}^{+}-V_{0}^{M}\right) & \left(1-\epsilon_{2}\right)\left(E_{2}^{+}-V_{0}^{M}\right)
\end{array}\right)
$$

we have the PL system translate to the matrix form

$$
\begin{gathered}
\max H(\mathcal{G})=\epsilon^{T} \mathcal{G} \\
\Theta \mathcal{G} \leq \Upsilon \\
\mathcal{G} \geq \mathcal{O} .
\end{gathered}
$$

\section{FUZZY BACKGROUND FOR THE ELECTRIC CIRCUIT MODEL}

In this section, we sumarize the relevant concepts of fuzzy sets of type-1, and fuzzy sets of type-2, which details can be found in $[2,5,6]$. Given a universe set, $X$, the set

$$
A=\left\{\left(x, \mu_{A}(x)\right), x \in X\right\}
$$

where $\mu_{A}$ a function is a fuzzy set of type- 1 over $X$, corresponding to the membership function $\mu_{A}$. The assembly of all fuzzy sets of type- 1 is denoted by $F(X)$. Given a number $\alpha \in[0,1]$, the $\alpha$-level of the fuzzy set $A$ is the set defined by

$$
[A]^{\alpha}=\left\{x \in X, \mu_{A}(x) \geq \alpha\right\} .
$$

Considering the set

$$
\operatorname{supp}(A)=\overline{\left\{x \in X, \mu_{A}(x)>0\right\}},
$$

which is, by definition, the support of the set $A$, the zero level of $A$ is the closure of $\operatorname{supp}(A)$, denoted by $\overline{\operatorname{supp}(A)}$.

Any fuzzy set can be regarded as a family of fuzzy sets. This is the essence of an identity principle known as the representation theorem. The representation theorem states that any fuzzy set $A$ can be decomposed into a series of its $\alpha$-level [7].

The fuzzy set of type-1, $A$, is called a fuzzy number when $X=\mathbb{R}$, there is $x \in X$ such that $\mu_{A}(x)=1$, all $\alpha$-levels are not empty and are closed intervals, and $A$ has bounded support. In this study is considered the fuzzy numbers defined as singleton of type-1, given through the membership function

$$
\mu_{r}(x)= \begin{cases}1 & \text { if } x=r \\ 0, & \text { if } x \neq r\end{cases}
$$

known as the characteristic function of the set $\{r\}$. We denote this fuzzy number by $\bar{r}$. 
Another type of fuzzy number considered is the trapezoidal fuzzy number defined by the membership function

$$
\mu_{A}(x)=\left\{\begin{array}{l}
\frac{x-a}{m-a}, \text { if } x \in[a, m] \\
1, \text { if } x \in[m, n] \\
\frac{b-x}{b-n}, \text { if } x \in[n, b] \\
0, \text { otherwise, }
\end{array}\right.
$$

denoted by $A=(a, m, n, b)$. The interval $[m, n]$ is known as the kernel of the trapezoidal fuzzy number $A$.

A fuzzy set of type-2, $\widetilde{A}$, on $X$ is the set

$$
\widetilde{A}=\left\{(x, u) ; \mu_{\widetilde{A}}((x, u)),(x, u) \in X \times[0,1]\right\},
$$

where $\mu_{\widetilde{A}}: X \times[0,1] \rightarrow[0,1]$ is the associated membership of the fuzzy set $\widetilde{A}$. The secondary membership function of $x^{\prime} \in X$ is the fuzzy set of type- 1 which universe is the set

$$
U_{x^{\prime}}=\overline{\left\{u \in[0,1], \mu_{\tilde{A}}\left(x^{\prime}, u\right)>0\right\}}
$$

and its membership is giving by $\mu_{\widetilde{A}}\left(x^{\prime}, u\right)$, with $u \in U_{x^{\prime}}$. Geometrically, this fuzzy set is obtained by the vertical cut with the parallel plane to the axis $u$ passing through $x=x^{\prime}$.

The so called superior primary membership function of type- 1 denoted by $\bar{\mu}_{\widetilde{A}}(x)$, and the inferior primary membership function of type-1, $\underline{\underline{\mu}} \widetilde{A}(x), x \in X$, are defined by

$$
\begin{aligned}
& \bar{\mu}_{\widetilde{A}}(x)=\sup \left\{u \in[0,1], \mu_{\widetilde{A}}(x, u)>0\right\}, \\
& \underline{\mu}_{\widetilde{A}}(x)=\inf \left\{u \in[0,1], \mu_{\widetilde{A}}(x, u)>0\right\} .
\end{aligned}
$$

A fuzzy set of type-2 that verifies $\mu_{\widetilde{A}}((x, u))=1$ for all $(x, u) \in X \times[0,1]$ is called an interval fuzzy set of type- 2 . When these type of sets have $\underline{\mu}_{\widetilde{A}}=\bar{\mu}_{\widetilde{A}}(x)$ for all $x \in X$, known as singleton of type-2.

We define the operation of addition between trapezoidal fuzzy numbers. Indeed, let $A_{i}=$ $\left(a_{i}, m_{i}, n_{i}, b_{i}\right), i=1,2$, two trapezoidal fuzzy numbers. By definition, we have

$$
A_{1}+A_{2}=\left(a_{1}+a_{2}, m_{1}+m_{2}, n_{1}+n_{2}, b_{1}+b_{2}\right) .
$$

An external product given $\alpha \in \mathbb{R}^{+}$is defined by

$$
\alpha \cdot A_{1}=\alpha\left(a_{1}, m_{1}, n_{1}, b_{1}\right)=\left(\alpha \cdot a_{1}, \alpha \cdot m_{1}, \alpha \cdot n_{1}, \alpha \cdot b_{1}\right) .
$$

In the case that $\alpha \in \mathbb{R}^{-}$, the definition is

$$
\alpha \cdot A_{1}=\alpha\left(a_{1}, m_{1}, n_{1}, b_{1}\right)=\left(\alpha \cdot b_{1}, \alpha \cdot n_{1}, \alpha \cdot m_{1}, \alpha \cdot a_{1}\right)
$$


With these definitions, the set of trapezoidal fuzzy numbers is a vector subspace of $F(\mathbb{R})$, which we denote by $\operatorname{Trap}(\mathbb{R})$.

Several proposals for an order relation, not necessarily a total order, over the set $\operatorname{Trap}(\mathbb{R})$ can be found in the literature $[1,4,8]$. In this study, it is proposed a novel total order in a particular vector subspace of $\operatorname{Trap}(\mathbb{R})$, composed by the fuzzy numbers

$$
\{A=(a, a, n, b), a, n, b \in \mathbb{R}\} .
$$

The set described in (3.3) it is denoted as $\operatorname{Trap}_{\mathrm{Right}}(\mathbb{R})$. It is easy to proved that the set $\operatorname{Trap}_{\text {Right }}(\mathbb{R})$ is, in fact, a subspace of $\operatorname{Trap}(\mathbb{R})$.

Let $A \in \operatorname{Trap}$ Right $(\mathbb{R})$, the new total order relation is constructed through the so call defuzzification function associated to the order [1], given by

$$
g_{\gamma}(A)=b+\gamma(n-b), \gamma \in[0,1],
$$

where $\gamma$ is a parameter in the interval $[0,1]$ that we defined as parameter of defuzzification of $g$. Defining a equivalence relation in $\operatorname{Trap} \operatorname{Right}(\mathbb{R})$ as

$$
A \text { is equivalent to } B \text { if and only if } g(A)=g(B) \text {, for all } A, B \in \operatorname{Trap}_{\text {Right }}(\mathbb{R}) \text {, }
$$

and the order relation

$$
A \text { is related to } B \text { if and only if } g(A)>g(B) \text {, for all } A, B \in \operatorname{Trap}_{\text {Right }}(\mathbb{R}),
$$

it is clear that this relation is a total order (3.6) upon Trap Right $(\mathbb{R})$, along with the equivalence relation (3.5), determines a total order in this set, denoted by “ $\succeq$ ”. The total order (3.6) is used in next section in order to transform a fuzzy linear programming in a classical one to obtain a fuzzy solution of the model (2.5).

\section{FUZZY LINEAR PROGRAMMING METHODS FOR THE ELECTRICAL CIRCUIT MODEL}

In this section, we consider the LP problem described in Section 2, which is given by the matrix form (2.5). The objective to use the FLP approach is to allow a constraint violation. This extension of the model is made through the coefficients of the objective function, and the constraints, using fuzzy numbers for its coefficients. Therefore, we consider the elements of LP components $H, \Theta$, and $\Upsilon$ in three cases:

1. real numbers (classical LP). In order to validate the proposed methods;

2. fuzzy numbers (FLP of type-1). The coefficients of $H$, and the entries of $\Theta$ are elements of $\operatorname{Trap}_{\text {Right }}(\mathbb{R})$. The vector $\Upsilon=\left(I_{0}^{M}, I_{0}^{m}\right)$, where $I_{0}^{M}$ and $I_{0}^{m} \in \mathbb{R}$;

3. fuzzy sets of type-2 (FLP of type-2). The coefficients of $H$ and the entries of $\Theta$ are singleton of type-2, whose projections in the plane $(x, u)$ belongs to $\operatorname{Trap} \operatorname{Right}(\mathbb{R})$. 
The first component of the vector $\Upsilon$ is a fuzzy set of type- 2 defined by the membership function

$$
\mu_{I_{0}^{m}}(x, u)=\left\{\begin{array}{l}
\alpha, \text { if } x \in\left[I_{0}^{m}-\delta_{L}, I_{0}^{m}\right], \\
u_{x}=\frac{x-I_{0}^{m}+\delta_{L}(1-\alpha)}{\delta_{L}(1-\alpha)-I_{0}^{m}}, \text { for } \alpha \in[0,1],
\end{array}\right.
$$

where $I_{0}^{m}-\delta_{L}$ is the minimum of level zero.

The second component of the vector $\Upsilon$ is a fuzzy set of type- 2 defined by the membership function

$$
\mu_{I_{0}^{M}}(x, u)=\left\{\begin{array}{l}
\alpha, \text { if } x \in\left[I_{0}^{M}, I_{0}^{M}+\delta_{R}\right], \\
u_{x}=\frac{I_{0}^{M}+\delta_{R}(1-\alpha)-x}{I_{0}^{M}+\delta_{R}(1-\alpha)}, \text { for } \alpha \in[0,1],
\end{array}\right.
$$

where $I_{0}^{M}+\delta_{R}$ is the maximum of level zero.

The graphics of these fuzzy sets of type- 2 are shown in Figure 2 for the independent constraint $I_{0}^{m}$, and in Figure 3 for $I_{0}^{M}$.

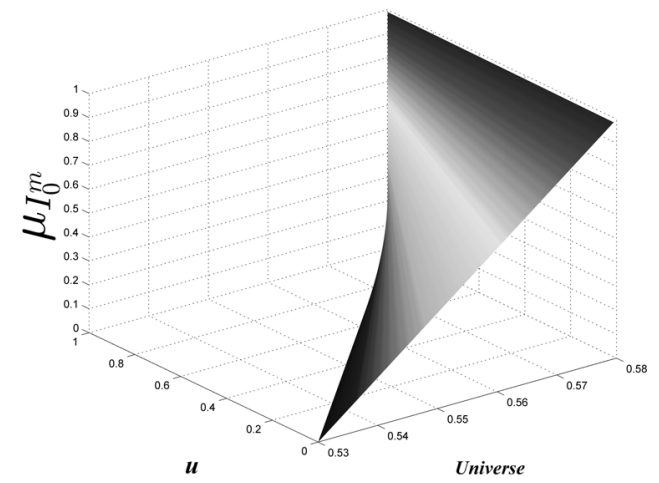

Figure 2: The fuzzy set of type-2 corresponding to the independent term of the second constraint.

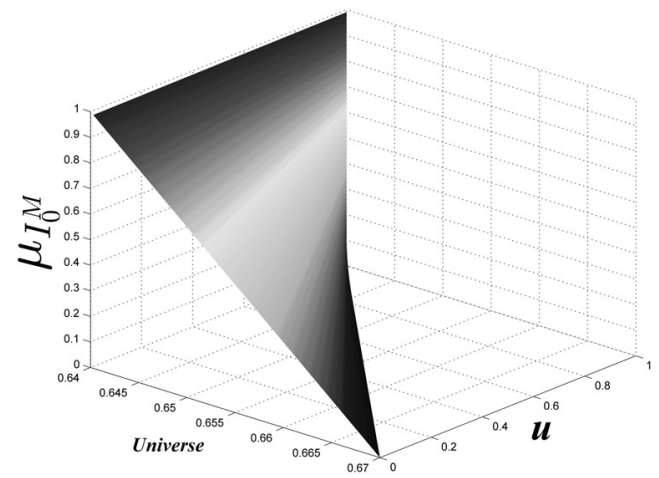

Figure 3: The fuzzy set of type-2 corresponding to the independent term of the first constraint.

The motivation to study the third case comes again, to allow a constraint violation in terms of the interval $\left[I_{0}^{m}, I_{0}^{M}\right]$. Extending this interval to $\left[I_{0}^{m}-\delta_{L}, I_{0}^{M}+\delta_{R}\right]$, for chosen values of $\delta_{L}$ and $\delta_{R}$ for which the PL has solution, induces to a feasible region, that we called the fuzzy feasible region, to be included in the crisp feasible region. Indeed, the PL constraint associated to the fuzzy feasible region is given by

$$
\begin{aligned}
& \left(1-\epsilon_{1}\right)\left(E_{1}^{-}-V_{0}^{m}\right) G_{1}+\left(1+\epsilon_{2}\right)\left(E_{2}^{-}-V_{0}^{m}\right) G_{2} \geq I_{0}^{M}+\delta_{R} \\
& \left(1+\epsilon_{1}\right)\left(E_{1}^{+}-V_{0}^{M}\right) G_{1}+\left(1-\epsilon_{2}\right)\left(E_{2}^{+}-V_{0}^{M}\right) G_{2} \leq I_{0}^{m}-\delta_{L}
\end{aligned}
$$




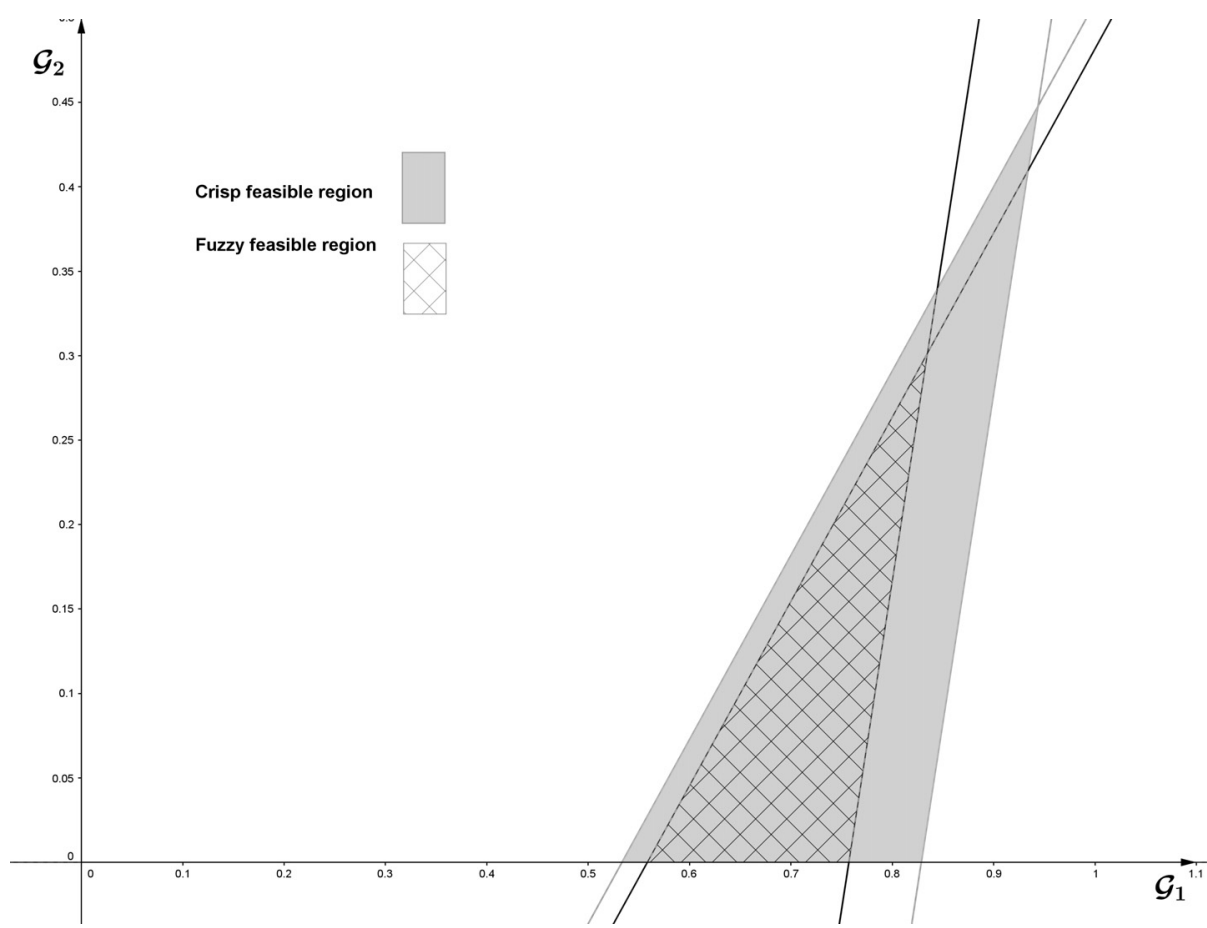

Figure 4: The crisp and fuzzy feasible regions for the values $\delta_{L}=0.03$ and $\delta_{R}=0.05$.

which yields in the set shown in Figure 4.

The extreme values, for which the PL has solution, $\delta_{L}$ and $\delta_{R}$ are

$$
0 \leq \delta_{L}<0.335 \text { and } 0 \leq \delta_{R}<0.207
$$

For the numerical simulations of Section 5, the values chosen are $\delta_{L}=0.03$ and $\delta_{R}=0.05$.

On the order hand, the resolution method applied in the case of the classical LP is the interior point [12], using the linear programming algorithm linprog of the software Matlab ${ }^{\circledR}$.

For case 2, it is applied the defuzzification order $g_{\gamma}$ on the fuzzy coefficients of the objective function, and the constraints, in order to calculate the optimum point through the case 1 method.

For case 3 , it is defined a parametric family indexed by $\alpha$ of PL problems of type-1, obtained by the cuts of the vector $\Upsilon$ at the $\alpha$-level. It is solved for each $\alpha$ a PL problem of type-1, the method used in case 2 . The solution is obtained by applying the representation theorem of $\alpha$-levels of the sets of type-2 [6].

\section{NUMERICAL RESULTS}

In the case of the classical linear programming, it is considered 


$$
H\left(\mathcal{G}_{1}, \mathcal{G}_{2}\right)=0.6 \mathcal{G}_{1}+0.9 \mathcal{G}_{2}, \quad \Theta=\left(\begin{array}{cc}
-1.2 & 1.1 \\
0.7 & -0.18
\end{array}\right) \quad \text { and } \quad \Upsilon=\left(\begin{array}{c}
-0.64 \\
0.58
\end{array}\right)
$$

where $\epsilon_{1}$ is $\pm 40 \%$, and $\epsilon_{2}$ is $\pm 10 \%$. The optimal solution obtained is $H(0.9437,0.4477)=$ 0.9691 .

For case 2, the components considered are:

$$
\begin{gathered}
H\left(\mathcal{G}_{1}, \mathcal{G}_{2}\right)=(0.4,0.6,1,1) \mathcal{G}_{1}+(0.6,0.9,1,1) \mathcal{G}_{2} \\
\Theta=\left(\begin{array}{cc}
(-2,-2,-1.2,-0.8) & (1,1,1.1,1.4) \\
(0.5,0.5,0.7,0.8) & (-0.2,-0.2,-0.18,-0.12)
\end{array}\right) \text { and } \Upsilon=\left(\overline{\frac{-0.64}{0.58}}\right) .
\end{gathered}
$$

The fuzzy trapezoidal numbers $\epsilon_{1}$ and $\epsilon_{2}$ used to construct the coefficients of the objective function $H$, and the entries of the matrix $\Theta$ are shown in Figure 5 and Figure 6.

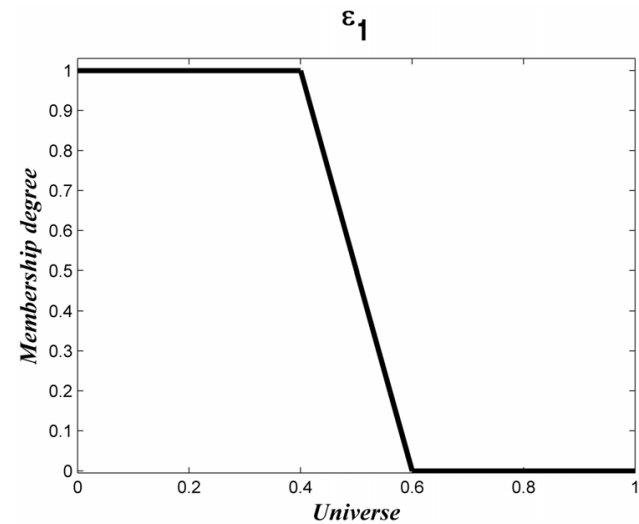

Figure 5: The fuzzy trapezoidal number $\epsilon_{1}$ used to construct the coefficients of the objective function $H$.

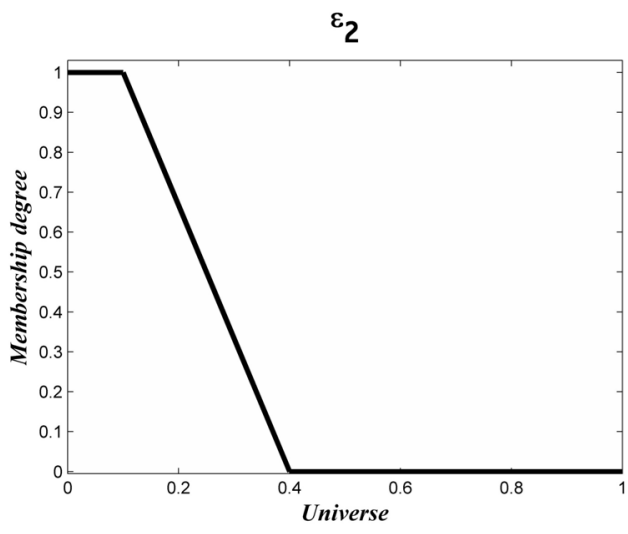

Figure 6: The fuzzy trapezoidal number $\epsilon_{2}$ used to construct the coefficients of the objective function $H$.

The graphics of the coefficients, $1-\epsilon_{1}$ and $1-\epsilon_{2}$ for the objective function $H$, are shown in Figure 7 and Figure 8.

The entries of the matrix $\Theta$ are shown in Figure 9 and Figure 10 (first row), and in Figure 11 and Figure 12 (second row).

We consider $\gamma \in[0.3,1]$ since that values lesser than 0.3 cause the linear programming to fail in encounter optimal solutions. For the value $\gamma=1$ we have $g_{\gamma}(A)=n$. As a consequence, the optimum point of the case 2 coincides with the case 1 , and the optimum value is the minimum of level 1 of optimum fuzzy number:

$$
H(0.9437,0.4477)=(0.5517,0.9691,1.3913,1.3913) .
$$

Other values of $\gamma$ yields in solutions that are shown in Figure 13. 


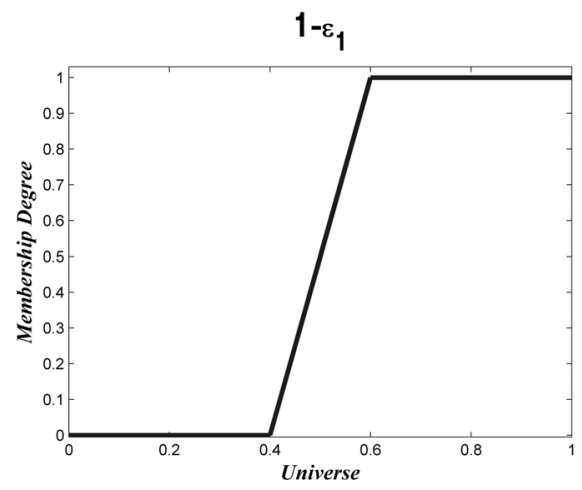

Figure 7: Coefficient $1-\epsilon_{1}$ of the objective function $H$.

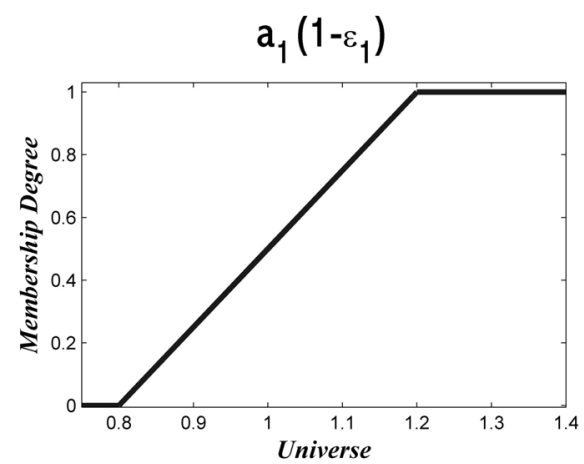

Figure 9: Entry $A_{11}$ of the matrix $\Theta$.

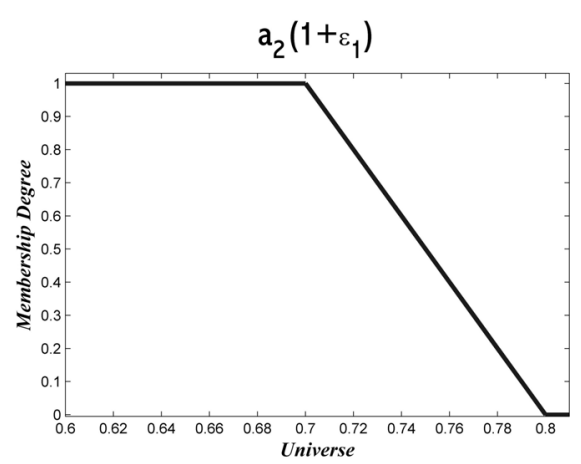

Figure 11: Entry $A_{21}$ of the matrix $\Theta$.

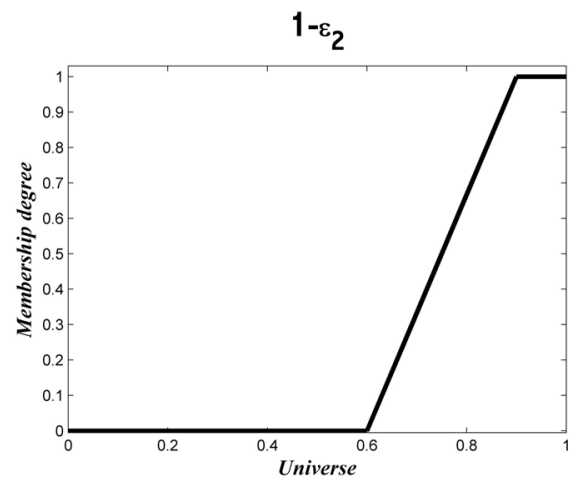

Figure 8: Coefficient $1-\epsilon_{2}$ of the objective function $H$.

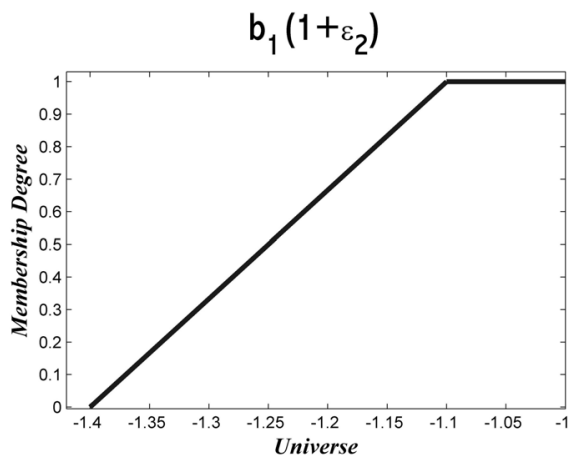

Figure 10: Entry $A_{12}$ of the matrix $\Theta$.

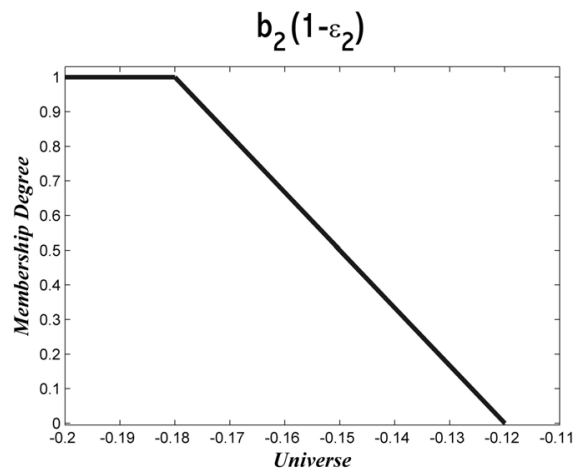

Figure 12: Entry $A_{22}$ of the matrix $\Theta$. 


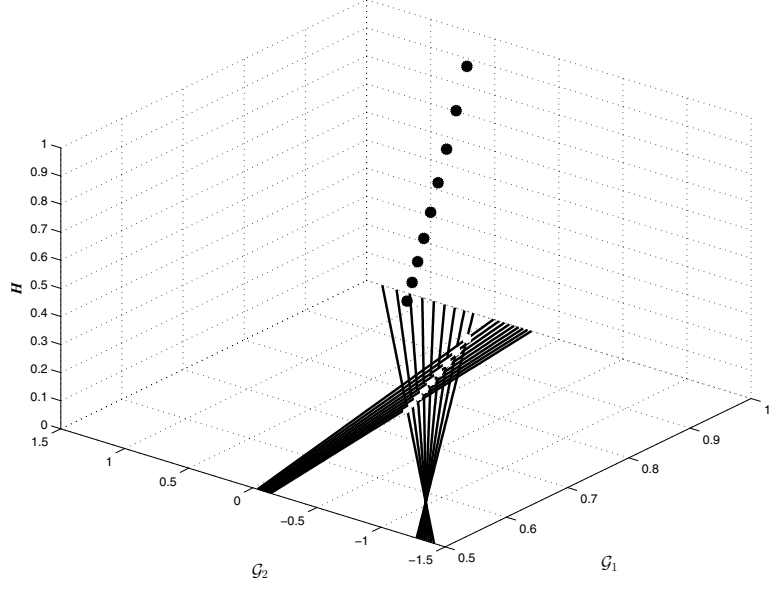

Figure 13: The optimal points corresponding to different values of $\gamma$.

For each $\gamma$, defuzzification parameter in the interval $[0.3,1]$, the optimum point is a fuzzy number of type-1, because for each level we get an optimum point (crisp), and a corresponding trapezoid optimal solution at this point. Thus, the optimum point has the fuzzy universe determined by the optimum level zero and level one, which in Figure 14 is represented by the black points. The membership function is the line determined by the points in space, whose first two coordinates are the coordinates of the optimum point level zero, and level one. The third coordinate is zero and one, respectively.

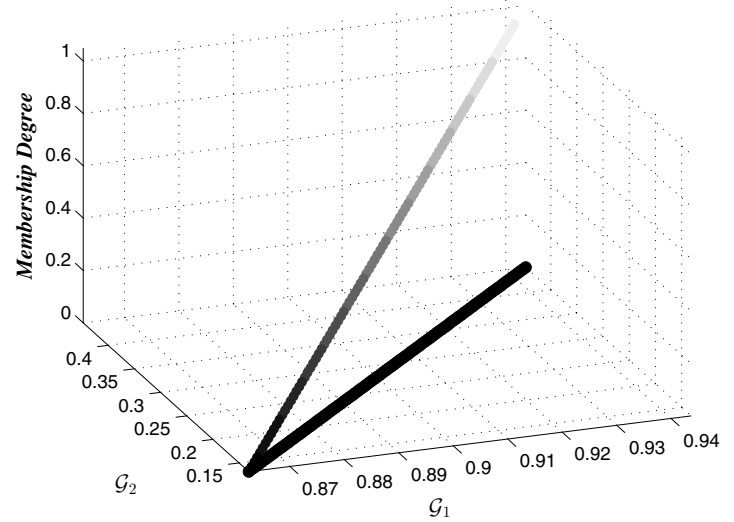

Figure 14: The optimal points and its membership degrees. 
In Figure 15, it is shown the graph of the optimal solution of type-2 obtained from each optimum point of Figure 14, and its corresponding optimal fuzzy solution. The gradient of gray colors in both the figures are corresponding and represent the levels from 0 to 1.

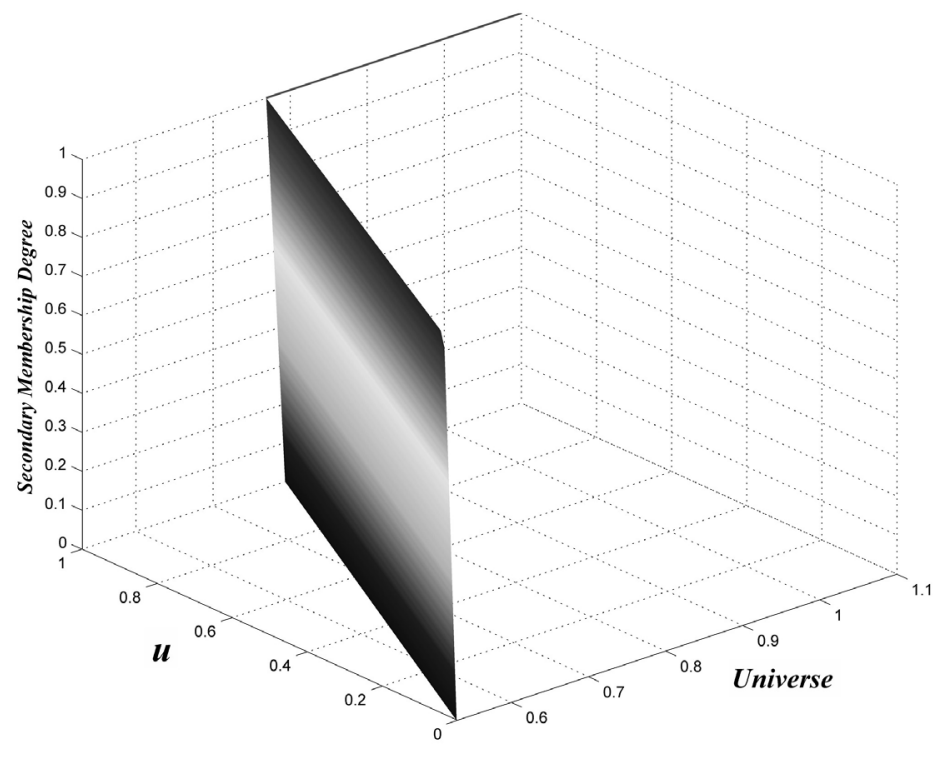

Figure 15: The fuzzy solution of type-2.

It is shown in Figure 16 the optimal points, and in Figure 17 the fuzzy solution of type- 2 for values of $\gamma$ in the interval $[0.7,1]$.

\section{CONCLUSION}

Emphasizing the existent uncertainty in the tolerances of an electrical circuit suggested by the device manufacturer, it is proposed in this study a fuzzy linear programming problem. In order to validate the results obtained, it is solved the classical linear programming problem. The optimal solution of fuzzy type- 1 is obtained using the an order through defuzzification that can transform the fuzzy problem into a classical linear programming. In order to build an optimal order, various defuzzification functions are defined over the subspace of oneside trapezoidal membership. The chosen order on these sets depends on real numbers between zero and one. Considering the defuzzification function corresponding to the parameters of defuzzification equal to one, it is obtained the same optimum point of the classical problem. Completing the study of a fuzzy linear programming model, the independent member of the constrains is considered as a fuzzy set of type-2. The solution is obtained by the representation theorem of $\alpha$-levels, solving, for each level, a linear programming problem of type-1. In this case, we use the same defuzzification function for ordering the subspace of oneside trapezoidal memberships with parameters near one, getting as optimum fuzzy point of relevance very close to the classic point. 


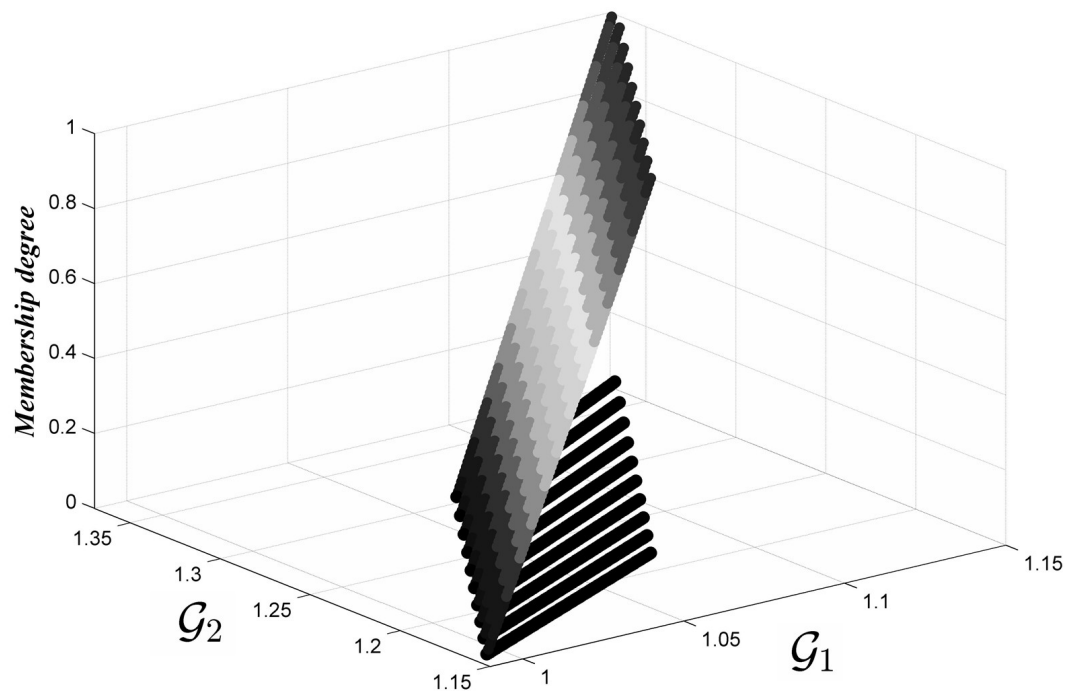

Figure 16: The optimal points corresponding to values of $\gamma \in[0.7,1]$, and its membership degrees.

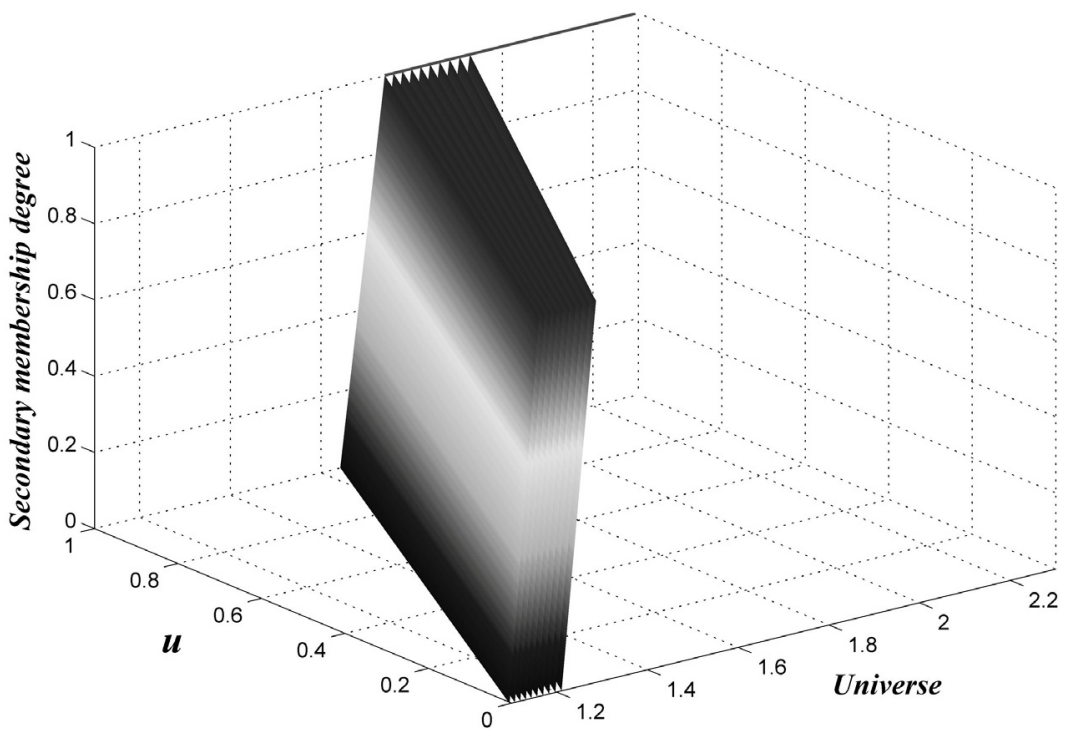

Figure 17: The fuzzy solution of type-2 corresponding to values of $\gamma \in[0.7,1]$.

RESUMO. Um problema de divisão de tensão de um circuito é modelado com o objetivo de determinar os valores centrados das resistências, de maneira que a impedância resistiva do divisor de tensão seja mínima. Este problema é equivalente a maximizar as admitâncias, associadas às resistências, que são definidas como a razão entre a corrente elétrica e sua vol- 
tagem, medida em Siemens. Três casos são analisados para os componentes de programação linear: números reais, números fuzzy do tipo 1 e conjuntos fuzzy do tipo 2 . O primeiro caso é considerado para a validação dos outros dois casos. A solução ótima na programação linear fuzzy do tipo 1 é obtida através de uma função de defuzzificação linear, definida no subespaço dos números fuzzy trapezoidais do espaço vetorial dos números fuzzy, o que permite resolver o correspondente problema de programação linear com componentes reais. Um estudo sobre o parâmetro para a defuzzificação linear é realizado para determinar o melhor representante da família de parâmetros. O teorema de representação dos $\alpha$-níveis é o método para obter a solução ótima do tipo 2. Para cada $\alpha$-nível é resolvido um problema de programação linear fuzzy do tipo 1 utilizando a metodologia anterior. Simulações numéricas ilustram os resultados nos três casos.

Palavras-chave: modelo de circuito elétrico, programação linear, programação linear fuzzy, conjuntos fuzzy do tipo 1 , conjuntos fuzzy do tipo 2 .

\section{REFERENCES}

[1] J.M. Adamo. Fuzzy decision trees. Fuzzy Sets and Systems, 4 (1980), 207-219.

[2] R.E. Bellman \& L.A. Zadeh. Decision-making in a fuzzy environment. Management Science, 17 (1973), 149-156.

[3] G.B. Dantzig. "Origins of the simplex method". Technical Report SOL 87-5, Department of Operations Research, Stanford University, Stanford, CA (1987).

[4] D. Dubois \& H. Prade. Operations on fuzzy numbers. International Journal of Systems Science, 9 (1978), 613-626.

[5] N.N. Karnik, J.M. Mendel \& Q. Liang. Type-2 fuzzy logic systems. IEEE Trans. on Fuzzy Systems, 7 (1999), 643-658.

[6] J.M. Mendel, M.R. Rajatia \& P. Sussner. On clarifying some definitions and notations used for type-2 fuzzy sets as well as some recommended changes. Information Sciences, 340-341 (2016), 337-345.

[7] W. Pedrycz \& F. Gomide. "An Introduction to Fuzzy Sets: Analysis and Design”. MIT Press, (1998).

[8] H. Prade, R.R. Yager \& D. Dubois (Editors). "Readings in Fuzzy Sets for Intelligent Systems". Morgan Kaufmann Publishers, (1993).

[9] N. Sahinidis. Optimization under uncertainty: state-of-the-art and opportunities. Computers and Chemical Engineering, 28 (2004), 971-983.

[10] J.J. Salazar González. Optimización matemática: Ejemplos y aplicaciones. Technical report Universidad de La Laguna, España, https : / / imarrero .webs . ull .es / sctm03 . v2 /, (2003).

[11] J.L. Verdegay. "Fuzzy Mathematical Programming". In: Fuzzy Information and Decision Processes, pp. 231-237, M.M. Gupta \& E. Sánchez Editors, (1982).

[12] J. Nocedal \& S.J. Wright. "Numerical Optimization". Springer-Verlag (2006). 